\title{
Frankfurter Radiologe beim German Medical Award ausgezeichnet
}

In Düsseldorf wurden zum vierten Mal die German Medical Awards an Ärzte, Forscher und Institutionen verliehen, die sich um fortschrittliche Patientenversorgung verdient gemacht haben. Prof. Dr. Thomas Vogl vom Universitätsklinikum Frankfurt erhält die Auszeichnung im Bereich Onkologie.

Der Medizin- und Gesundheitsmanagement-Preis prämiert herausragende Leis- tungen und Verfahren bei der Patientenversorgung. In verschiedenen Kategorien werden medizinische Innovationen, aber auch soziales Engagement geehrt. Schirmherr der Veranstaltung ist der nordrheinwestfälische Minister für Arbeit, Gesundheit und Soziales, Karl-Josef Laumann.

Prof. Thomas Vogl, Direktor des Instituts für Diagnostische und Interventionelle Radiologie am Universitätsklinikum Frankfurt, erhält den ersten Preis in der Kategorie „Medical Innovation Praxen und Kliniken: Onkologie“. Prof. Vogl wird für die Weiterentwicklung eines minimalinvasiven Verfahrens in der Krebstherapie geehrt, bei dem das Chemotherapeutikum mittels eines Katheters gezielt in das Tumorgewebe eingebracht wird. „Je passgenauer es angewendet wird, umso besser ist die therapeutische Effizienz und umso sicherer ist es für den Patienten“, lobt Prof. Matthias 


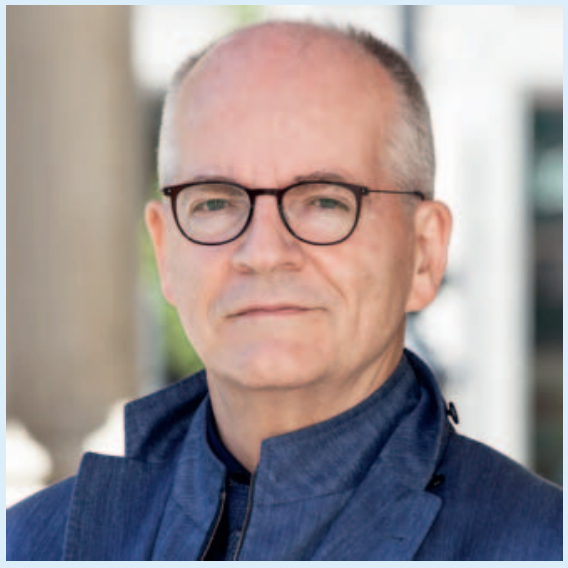

Prof. Dr. Thomas Vogl
Keidel, Chefarzt der Klinik für Akutneurologie in Bad Neustadt a.d. Saale, in seiner Laudatio die Leistungen des Preisträgers.

Die German Medical Awards sollten ursprünglich im Rahmen der medizinischen Fachmesse MEDICA am 17. November 2020 in Düsseldorf verliehen werden. Die Gala wurde aufgrund der SARS-CoV-2-Pandemie abgesagt und fand in diesem Jahr digital statt.

Weitere Informationen erhalten Sie beim Universitätsklinikum Frankfurt:

www.kgu.de 